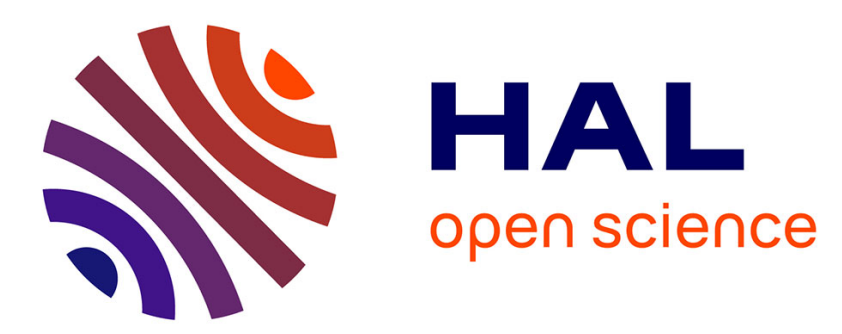

\title{
Modification du spectre d'absorption dans l'infrarouge lointain du phosphate dihydrogéno-ammonique lors de la transformation antiferro-électrique
}

\author{
F. Brehat, A. Hadni
}

\section{- To cite this version:}

F. Brehat, A. Hadni. Modification du spectre d'absorption dans l'infrarouge lointain du phosphate dihydrogéno-ammonique lors de la transformation antiferro-électrique. Revue de Physique Appliquée, 1974, 9 (2), pp.443-445. 10.1051/rphysap:0197400902044300 . jpa-00243799

HAL Id: jpa-00243799

https://hal.science/jpa-00243799

Submitted on 1 Jan 1974

HAL is a multi-disciplinary open access archive for the deposit and dissemination of scientific research documents, whether they are published or not. The documents may come from teaching and research institutions in France or abroad, or from public or private research centers.
L'archive ouverte pluridisciplinaire HAL, est destinée au dépôt et à la diffusion de documents scientifiques de niveau recherche, publiés ou non, émanant des établissements d'enseignement et de recherche français ou étrangers, des laboratoires publics ou privés. 


\author{
Classification \\ Physics Abstracts \\ 8.822

\section{MODIFICATION DU SPECTRE D'ABSORPTION DANS L'INFRAROUGE LOINTAIN DU PHOSPHATE DIHYDROGÉNO-AMMONIQUE LORS DE LA TRANSFORMATION ANTIFERRO-ÉLECTRIQUE}

\author{
F. BREHAT et A. HADNI \\ Université de Nancy I, France \\ (Reçu le 9 avril 1973)
}

\begin{abstract}
Résumé. - On a observé moins de bandes d'absorption de basses fréquences que la théorie des groupes n'en fait prévoir pour les vibrations externes du réseau. Toutefois, en ce qui concerne les vibrations normales du type $\mathrm{B}_{2}$ de la phase para-électrique, on en observe deux alors qu'on n'en prévoit qu'une. Il semble bien qu'il s'agisse là, comme dans $\mathrm{KH}_{2} \mathrm{PO}_{4}$, de l'effet du couplage des phonons avec le mouvement des protons. Par ailleurs, l'absorption augmente brutalement au passage de la phase antiferro-électrique à la phase para-électrique. Elle serait due à l'absorption induite par le désordre des protons [1] dans la phase para-électrique.

Abstract. - Fewer low frequency absorption bands than expected from group theory have been observed. However, in the case of $\mathrm{B}_{2}$-type vibrations in the paraelectric phase, two are observed whereas only one was expected. It seems that the doubling is due, as in the case of KDP, to a coupling of phonons with proton tunneling. We have also observed a sudden increase of absorption during the transition from the antiferroelectric phase to the paraelectric. It might be due to the proton-disorder-induced absorption [1].
\end{abstract}

On sait que le phosphate dihydrogéno-ammonique subit une transition de phase à $148 \mathrm{~K}$. Au-dessus, la maille élémentaire est quadratique ( $a=b=7,47 \AA$; $c=7,58 \AA$ à $300 \mathrm{~K})$, au-dessous elle devient orthorhombique ( $a=7,59 ; b=7,51 ; c=7,48 \AA$ à $143 \mathrm{~K})$ et donne une phase antiferro-électrique.

Le phosphate dihydrogéno-ammonique a déjà été étudié dans l'infrarouge lointain [1]. Il a été montré que lorsqu'on élève la température au-dessus de $148 \mathrm{~K}$ une absorption générale s'établit brusquement. Elle proviendrait d'un désordre plus grand dans la position des protons [1]. Elle est si grande qu'elle avait rendu impossible jusqu'à présent l'étude du spectre de transmission de la phase para-électrique.

Nous donnons ici des spectres de lames d'épaisseur inférieure à $10 \mu \mathrm{m}$, qui ont permis d'étudier la phase para-électrique. Il est alors possible de discuter de la modification du spectre au cours de la transition de phase. Par ailleurs, nous avons étendu le domaine spectral au-delà de $140 \mathrm{~cm}^{-1}$ jusqu'à $250 \mathrm{~cm}^{-1}$.

1. Expérimentation. - Le monocristal se prépare facilement par évaporation à température constante d'une solution saturée. Une lame taillée parallèlement au plan (010) est étudiée en lumière polarisée dans un spectromètre à réseau fonctionnant entre 10 et $250 \mathrm{~cm}^{-1}$, et muni d'un cryostat [2].

2. Résultats. - La figure 1 représente le spectre d'absorption lorsque le champ électrique de l'onde est parallèle à l'axe $c$ d'ordre 4 du cristal, la figure 2 correspond à la direction $a$ perpendiculaire à cet axe. Dans les 2 cas, l'absorption atteint plusieurs milliers de $\mathrm{Np} \mathrm{cm} \mathrm{cm}^{-1}$ à $300 \mathrm{~K}$ avec 2 bandes larges. A $80 \mathrm{~K}$ dans la phase antiferro-électrique, l'absorption générale a presque disparu dans la région des basses fréquences, et des raies assez fines apparaissent.

Le tableau I résume ces mesures. Les bandes trouvées à $300 \mathrm{~K}$ précisent heureusement les données de Yoshinaga et al. [3] sur les spectres de réflexion à $300 \mathrm{~K}$, avec une large bande centrée vers $220 \mathrm{~cm}^{-1}$ pour $\mathrm{E} / / c$, et une bande plus étroite centrée vers $175 \mathrm{~cm}^{-1}$ pour $\mathrm{E} / / a$. Les bandes décrites à $80 \mathrm{~K}$ confirment un travail précédent [1], et le complètent de 190 à $250 \mathrm{~cm}^{-1}$.

Nous avons essayé de suivre l'évolution de ces 


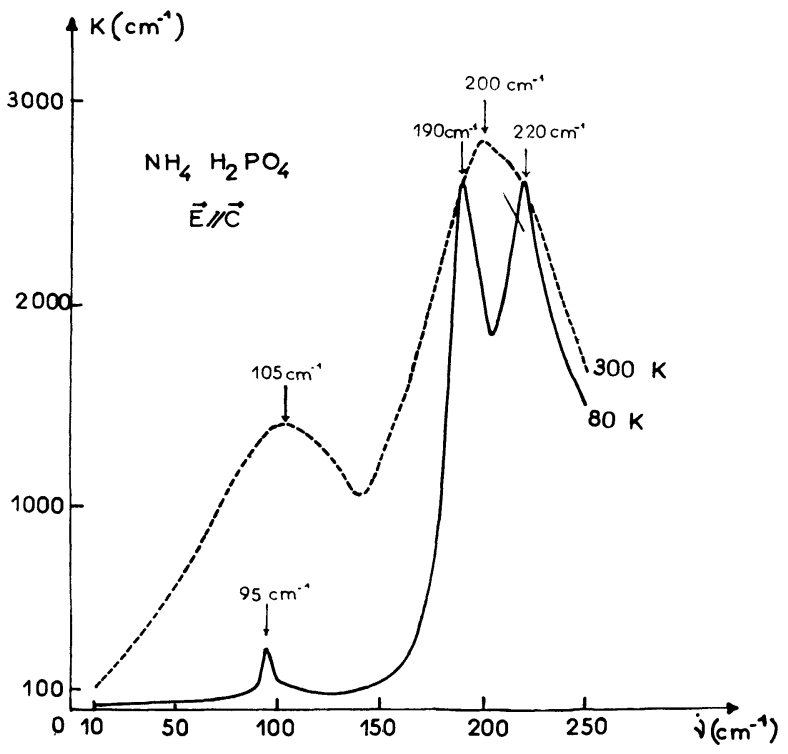

FIG. 1. - Coefficient d'absorption du phosphate dihydrogénoammonique de 10 à $250 \mathrm{~cm}^{-1}$, le champ électrique de l'onde incidente est parallèle à l'axe $c$ du cristal.

\section{TABLEAU I}

Bandes d'absorption du phosphate dihydrogénoammonique dans la phase para-électrique $(300 \mathrm{~K})$ et dans la phase antiferro-électrique $(80 \mathrm{~K})$. Une seule vibration $\mathrm{B}_{2}$ est prévue par le dénombrement

\begin{tabular}{|c|c|c|c|c|c|}
\hline \multicolumn{3}{|c|}{$300 \mathrm{~K}\left(\mathrm{D}_{2 \mathrm{~d}}\right)$} & \multicolumn{3}{|c|}{$80 \mathrm{~K}\left(\mathrm{D}_{2}\right)$} \\
\hline$/ / \mathbf{a}$ & $\mathbf{E} / / \mathbf{c}$ & Sym. & Sym. & $\mathbf{E} / / \mathbf{c}$ & $\mathbf{E} / / \mathbf{a}$ \\
\hline & $\begin{array}{r}105 \\
\left(\omega_{-}\right)\end{array}$ & $\mathrm{B}_{2}$ & $\begin{array}{l}\mathrm{B}_{1} \\
\mathrm{~B}_{1} \\
\mathrm{~B}_{1}\end{array}$ & $\begin{array}{c}95 \mathrm{~cm}^{-1} \\
99[1] \\
\text { non obs. }\end{array}$ & \\
\hline
\end{tabular}

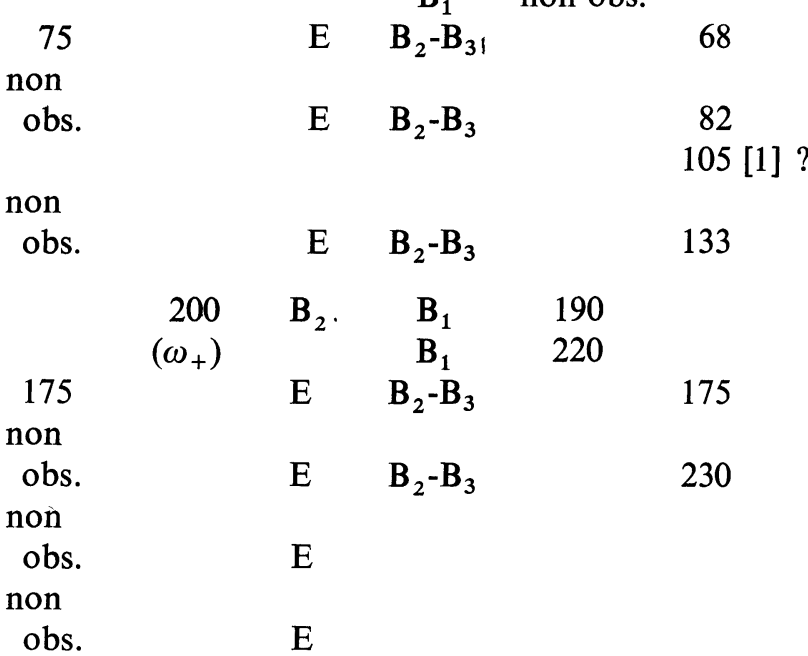

bandes en fonction de la température. Il semblerait [2] qu'entre $80 \mathrm{~K}$ et $148 \mathrm{~K}$ elles diminuent un peu d'intensité, sans s'élargir notablement, et qu'au-dessus du point de Curie, ce soient des bandes nouvelles incomparablement plus larges qui apparaissent. Par ailleurs,

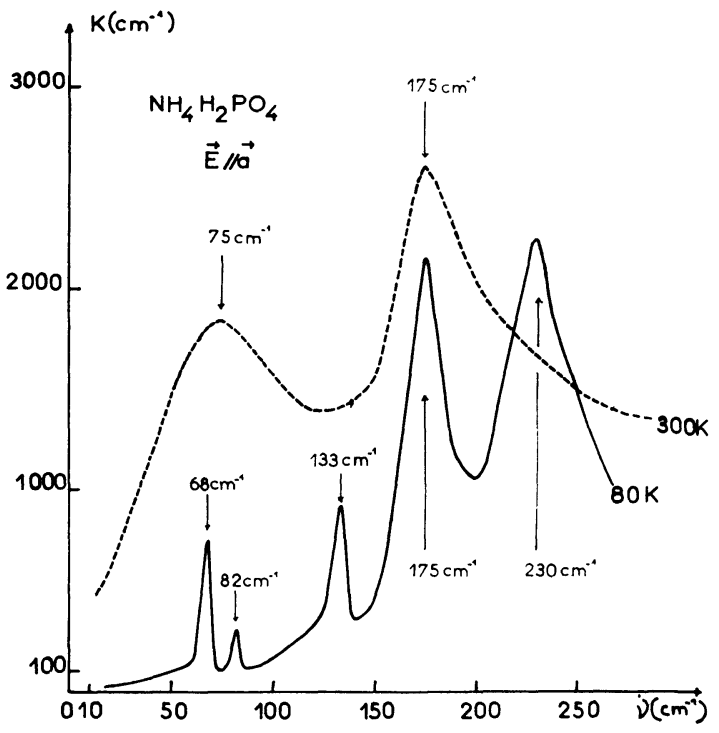

FIG. 2. - Coefficient d'absorption du phosphate dihydrogénoammonique de 10 à $250 \mathrm{~cm}^{-1}$, le champ électrique de l'onde incidente est perpendiculaire à l'axe $c$ du cristal.

les bandes observées à $300 \mathrm{~K}$ vers 105 et $75 \mathrm{~cm}^{-1}$ se déplacent légèrement vers les basses fréquences lorsque la température est abaissée vers la température de Curie [2].

3. Discussion. - Chaque unité $\mathrm{NH}_{4} \mathrm{H}_{2} \mathrm{PO}_{4}$ est formée d'un ion ammonium, et d'un ion complexe $\mathrm{H}_{2} \mathrm{PO}_{4}^{-}$que nous considérons comme formant un tout. Par ailleurs, dans la maille primitive du cristal, il $\mathrm{y}$ a 2 unités formulaires. Nous avons donc 4 ions complexes et nous attendons $3 \times 4-3=9$ modes normaux de translation de ces ions, et 12 modes de libration. On peut classer les 21 modes normaux de vibrations externes. Dans la phase para-électrique 8 modes sont actifs, 7 du type $E$ et 1 du type $B_{2}$. Dans la phase antiferro-électrique 15 modes normaux sont actifs, 5 de type $B_{1}$ donnant une variation de moment dipolaire $\mathrm{M}_{c}$ portée par l'axe $c, 5$ de type $\mathrm{B}_{2}\left(M_{b}\right)$ et 5 de type $\mathrm{B}_{3}\left(M_{a}\right)$.

Le tableau I montre que dans la phase para-électrique sur les 7 bandes du type $\mathrm{E}$ attendues, 2 seulement très larges apparaissent à 75 et $175 \mathrm{~cm}^{-1}$. Par ailleurs, au lieu d'une bande de type $B_{2}$, on en observe 2, comme dans le cas du dihydrogénophosphate de potassium [4] ou de rubidium [5]. On est conduit là aussi à fournir la même explication : un couplage entre les vibrations d'antitranslation des ions $\mathrm{PO}_{4}^{3-}$ et $\mathrm{NH}_{4}^{-}$, et le tunnelling des protons entre 2 puits de potentiel, soit en phase $\left(\omega_{+}\right)$, soit en opposition de phase $\left(\omega_{-}\right)$.

Dans la phase antiferro-électrique, sur les 5 vibrations attendues de type $B_{1}$, on en observe 4 à 95,99 , 190 et $220 \mathrm{~cm}^{-1}$. Mais pour les 5 vibrations de type $B_{3}$ et les 5 vibrations de type $\mathbf{B}_{2}$, on n'observe que 6 bandes d'absorption. On s'est étonné de ne pas trouver dans 
une lame taillée perpendiculairement à l'axe $c$, le dichroïsme qui devrait séparer les vibrations de type $B_{2}$ et $\mathbf{B}_{3}$ de la phase antiferro-électrique. En fait, lors du passage de la phase quadratique à la phase orthorhombique, le cristal se brise en petits domaines rectangulaires dont les côtés sont parallèles aux axes cristallographiques, donc aux lignes neutres. On a pu vérifier au microscope du Dr Poty. [6] qui permet de travailler à $100 \mathrm{~K}$, qu'il s'agissait bien des lignes neutres. Toutefois, d'un domaine à l'autre la ligne neutre peut être parallèle à l'axe $a$, puis à $b$. On comprend ainsi que la mosaïque à laquelle on aboutit ne présente ni dichroïsme, ni biréfringence.

Nos résultats confirment les études du phosphate diacide de potassium par diffusion Raman réalisées par Hammer [7] et Agrawal [8], comme nous Hammer a observé une augmentation du nombre des modes actifs dans la phase antiferro-électrique à $100 \mathrm{~K}$ [7].

Ces études nous permettent aussi de comparer les spectres d'absorption infrarouge des "phosphates diacides de potassium et de rubidium [4], [5] et d'ammonium, aux spectres de diffusion Raman de ces mêmes composés [7], [8]. A la température ordinaire, ces cristaux ont la même structure, parmi les 5 classes de vibration, seules les classes $B_{2}$ et $E$ sont actives à la fois en infrarouge et en Raman et permettent la comparaison des différents spectres. A basse température, la structure antiferro-électrique du phosphate diacide d'ammonium est différente de la structure ferro-électrique des 2 autres composés.

Dans la phase para-électrique à $300 \mathrm{~K}$, on observe une correspondance remarquable des spectres de vibration de la classe $B_{2}, 2$ modes de basse fréquence sont actifs en absorption infrarouge et en diffusion Raman dans chacun des 3 composés [4], [5], [7], [8], les fréquences sont du même ordre de grandeur, celles des modes de ADP étant les plus élevées. Nous avons attribué ces modes au couplage, en phase et en opposition de phase, du mouvement d'antitranslation des ions lourds et du mouvement des protons entre 2 puits de potentiel [4], [5]. Cependant nous n'observons que 2 modes $E$ actifs en infrarouge [3] dans ADP alors que 4 le sont en diffusion Raman [8].

Dans la phase de basse température à $80 \mathrm{~K}$, nous retrouvons une bonne correspondance des différents spectres d'absorption infrarouge et de diffusion Raman de ADP, KDP et RbDP à un déplacement près des fréquences, bien que la structure de ADP dans cette phase soit différente de celle de KDP et RbDP [5], [7], [8]. Il existe une analogie entre les modes $B_{1}$ de ADP et les modes $A_{1}$ de KDP et RbDP. Pour les modes $B_{2}$ et $B_{3}$ de $A D P$, nous retrouvons l'absence de dichroïsme déjà observée pour KDP et $\mathrm{RbDP}$ dans les classes $\mathrm{B}_{1}$ et $\mathrm{B}_{2}$ à $80 \mathrm{~K}$ [5]. Les modes -actifs en infrarouge dans ces classes ont des fréquences voisines de celles qu'ont observées Hammer et Agrawal en diffusion Raman tant pour ADP [7] que pour KDP [7], [8] et RbDP [7]. Nous pouvons attribuer ces modes à des modes de réseau, le déplacement des fréquences étant dû à la variation de la masse du cation.

4. Conclusion. - Le dénombrement des vibrations externes du phosphate dihydrogéno-ammonique, avec 2 unités formulaires $\mathrm{NH}_{4} \mathrm{H}_{2} \mathrm{PO}_{4}$ dans la maille élémentaire, fait prévoir dans la phase para-électrique 8 vibrations actives en infrarouge : 7 vibrations de type $E$ polarisées perpendiculairement à l'axe quadratique $c$, et 1 vibration du type $B_{2}$ polarisée parallèlement. On n'observe que 2 bandes larges du type $\mathrm{E}$, mais par contre 2 aussi du type $B_{2}$ à 75 et $175 \mathrm{~cm}^{-1}$, alors qu'on n'en attend qu'une. Par analogie avec ce qui avait été vu pour $\mathrm{KH}_{2} \mathrm{PO}_{4}$ et $\mathrm{RbH}_{2} \mathrm{PO}_{4}$, on suggère un couplage de la vibration du type $\mathrm{B}_{2}$ avec le passage des protons par effet tunnel entre les deux puits de leur potentiel qui donnerait 2 fréquences $\omega_{-}$et $\omega_{+}$.

Dans la phase antiferro-électrique, on attend 5 vibrations du type $B_{1}$ polarisées parallèlement à la direction $c, 5$ vibrations $B_{2}$ et 5 vibrations $B_{3}$ polarisées parallèlement aux directions $a$ et $b$. On observe 4 des vibrations $B_{1}$, et seulement 6 des vibrations $B_{2}$ et $B_{3}$ sur un total de 10 attendues.

\section{Bibliographie}

[1] Morlot, G., Villermain-Lecollier, G. et Hadni, A., Phys. Stat. Sol. (b) 49 (1972) K 47.

[2] Brehat, F., thèse Nancy, 1972.

[3] Kawamura, T., Mitsuishi, A. et Yoshinaga, H., J. Phys. Soc. Japan 28 (1970) 227.

[4] Brehat, F. et Hadni, A., C. R. Hebd. Séan. Acad. Sci. 271 (1970) 1137.
[5] Brehat, F., Hadni, A., Aubry, J. et Zanne, M., Rev. Phys. Appl. 7 (1972) 163.

[6] Docteur Poty, Centre de Recherches Pétrographiques et Géochimiques, 54-Vandœuvre-les-Nancy.

[7] Hammer, H., Light Scattering in Solids (M. Balkanski éditeur, Paris) 1971 p. 425.

[8] Agrawal, D. K. and Perry, C. H., Light Scattering in Solids (M. Balkanski éditeur, Paris) 1971 p. 429. 